\title{
Nonperiodicity Implies Unique Composition for Self-Similar Translationally Finite Tilings*
}

\section{B. Solomyak}

Department of Mathematics, Box 354350, University of Washington,

Seattle, WA 98195-4350, USA

solomyak@math.washington.edu

\begin{abstract}
Let $\mathcal{T}$ be a translationally finite self-similar tiling of $\mathbf{R}^{d}$. We prove that if $\mathcal{T}$ is nonperiodic, then it has the unique composition property. More generally, $\mathcal{T}$ has the unique composition property modulo the group of its translation symmetries.
\end{abstract}

\section{Introduction}

We consider tilings of the Euclidean space $\mathbf{R}^{d}$. A tile is a compact set which is the closure of its interior. A tiling is a collection of tiles with disjoint interiors whose union is the whole space. (This may seem too general but it is the right setting for our results. In first reading, however, one can think of polyhedral tiles.) A finite set of tiles with disjoint interiors is called a patch. We always assume that our tiling is translationally finite which means that, for any $R>0$, it has finitely many patches of diameter less than $R$, up to translation. In particular, there are finitely many tiles up to translation. Two tilings $\mathcal{T}_{1}$ and $\mathcal{T}_{2}$ are said to be locally isomorphic if every patch of $\mathcal{T}_{1}$ can be found in $\mathcal{T}_{2}$ and vice versa (again, the patches are identified by translations). Local isomorphism is an equivalence relation; the equivalence classes are called LI classes, or species.

A vector $x \in \mathbf{R}^{d}$ is a period of the tiling $\mathcal{T}$ if $\mathcal{T}+x=\mathcal{T}$. A tiling is called crystallographic [LP] if it has $d$ linearly independent periods, and nonperiodic if it has no nonzero periods. As the term suggests, crystallographic tilings have been used to model crystals. In the last decade, nonperiodic tilings have attracted much attention, largely motivated by the discovery of quasi-crystals in 1984 . Among nonperiodic tilings, the best known is the Penrose tiling (or rather, the whole LI class which consists of

\footnotetext{
* This research was partially supported by NSF.
} 


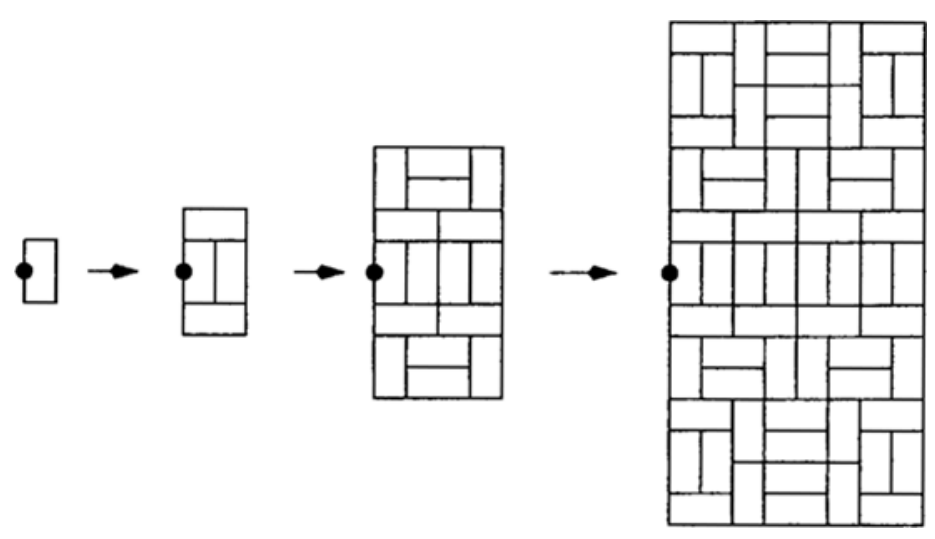

Fig. 1. A self-similar domino tiling.

uncountably many tilings), see [GS], which turned out to be a good model for certain quasi-crystallographic alloys.

One of the many remarkable properties of the Penrose tiling is self-similarity: its tiles can be grouped into patches to form a new tiling that is locally isomorphic to the original one dilated by the golden ratio (called the expansion constant). This procedure is called a composition. (The statement about the Penrose tiling is literally true if the "Robinson triangles" are used as tiles; in the case of "kites and darts" or "thick and thin rombi" a slight modification is needed; see [GS].) This property (with some expansion constant, or more generally, some expansive similarity) is shared by a large class of tilings which we call self-similar tilings.

We say that a self-similar tiling has the unique composition property if the composition procedure is unique. Closely related notions have been considered under the names uniquely hierarchical and inflation-deflation symmetry. The unique composition property can be illustrated by the following examples.

Example 1. Let $\mathcal{T}$ be the tiling of the plane by squares $\left\{[0,1]^{2}+(m, n): m, n \in \mathbf{Z}\right\}$. of course, this is a crystallographic tiling. It is self-similar with expansion constant 2 , but does not have the unique composition property, since there are four distinct ways to compose its tiles into a tiling which is a translate of $2 \mathcal{T}$.

Example 2. A tiling of the plane by "dominoes" $2 \times 1$ and $1 \times 2$ is shown in Fig. 1 . The origin is indicated by a dot, and the arrows represent a "substitution" procedure consisting of multiplication by 2 and subdivion. The increasing patches "converge" to a tiling of the half-plane; the other half can be tiled by mirror symmetry. We get a selfsimilar tiling of the plane with expansion constant 2 . It is easy to see directly that the unique composition property holds in this case.

A well-known "folklore" theorem (see Theorem 10.1.1 of [GS]) states that a tiling with the unique composition property is nonperiodic. Our main result is the converse: 
A nonperiodic translationally finite self-similar tiling has the unique composition property.

The unique composition property is often useful. In particular, Anderson and Putnam [AP] assumed it in their study of the $K$-theory of $C^{*}$-algebras arising from self-similar tilings. In [So] we used unique composition to construct eigenfunctions for tiling dynamical systems.

Unique composition is a tiling analog of "bilateral recognizability," a property of symbolic substitution systems. Mossé [M] proved that a nonperiodic primitive substitution always has this property, and our proof is modeled after her argument.

Two variations are also considered in this paper. First, let $\mathcal{K}=\left\{x \in \mathbf{R}^{d}: \mathcal{T}+x=\mathcal{T}\right\}$ be the group of translation symmetries for a self-similar tiling $\mathcal{T}$. We show that if $\mathcal{U}_{1}$ and $\mathcal{U}_{2}$ are two tilings composed from the tiles of $\mathcal{T}$, both locally isomorphic to the inflation of $\mathcal{T}$, then $\mathcal{U}_{2}=\mathcal{U}_{1}+x$ for some $x \in \mathcal{K}$. This formulation covers nonperiodic tilings as well, when $\mathcal{K}=\{0\}$. Second, we extend the results to self-affine tilings, when the inflation map is an arbitrary expansive linear transformation of $\mathbf{R}^{d}$.

\section{Definitions and Statement of Results}

Suppose that there is a finite set of tiles $\mathcal{A}=\left\{A_{1}, \ldots, A_{m}\right\}$, called prototiles, and denote by $\mathcal{X}_{\mathcal{A}}$ the set of tilings all of whose tiles are translates of the $A_{i}$. Sometimes it is convenient to have distinct prototiles which are translates of one another. Then we assign "markings" to distinguish them, and a tile should be thought of as a pair of a set and a marking. Two tiles are considered to be equal, $T=T^{\prime}$, if they coincide as sets and have the same marking. If $T$ is a tile, then $T+x$ denotes its translate by a vector $x \in \mathbf{R}^{d}$, with the same marking.

A patch is a finite set of tiles with disjoint interiors. The support of a patch $P$, denoted by $\operatorname{supp}(P)$, is the union of tiles in $P$. The translate of $P$ by $x \in \mathbf{R}^{d}$ is $P+x=\{T+x$ : $T \in P$ ). We say that $P$ is a $\mathcal{T}$-patch if $P \subset \mathcal{T}$. The diameter of the support of the patch $P$ is denoted by $\operatorname{diam}(P)$. We always assume that the tiling is translationally finite:

$$
\begin{gathered}
\forall R>0, \quad \text { the number of } \mathcal{T} \text {-patches } P, \text { having } \operatorname{diam}(P) \leq R, \\
\text { is finite, up to translation. }
\end{gathered}
$$

Next we define self-affine and self-similar tilings. Let $\varphi$ be a linear map on $\mathbf{R}^{d}$. It is called expansive if,

$$
\forall x \in \mathbf{R}^{d}, \quad\|\varphi x\| \geq \lambda_{0}\|x\| \quad \text { for some } \quad \lambda_{0}>1 .
$$

The map $\varphi$ is a similarity if $\|\varphi(x)\|=\lambda\|x\|$ for all $x$.

Suppose that there is a substitution rule $\omega$ on the set of prototiles $\mathcal{A}$ that associates to each $A_{i}$ a patch supported on $\varphi A_{i}$. More precisely, there exist $n_{i j} \geq 0$ and $x_{i j k} \in \mathbf{R}^{d}$ for $1 \leq i, j \leq m ; 1 \leq k \leq n_{j}$, such that

$$
\omega\left(A_{i}\right)=\left\{A_{j}+x_{i j k}: 1 \leq j \leq m, 1 \leq k \leq n_{i j}\right\} \quad \text { for } \quad 1 \leq i \leq m,
$$


with

$$
\varphi A_{i}=\bigcup_{j=1}^{m} \bigcup_{k=1}^{n_{i j}}\left(A_{j}+x_{i j k}\right) .
$$

Here all the $A_{j}+x_{i j k}$ must have disjoint interiors; if some $n_{i j}=0$ then the corresponding union $\bigcup_{k=1}^{n_{i j}}\left(A_{j}+x_{i j k}\right)$ is considered to be empty.

The substitution (2) is extended to all tiles by $\omega\left(A_{i}+x\right)=\omega\left(A_{i}\right)+\varphi x$, and to patches and tilings by $\omega(P)=\bigcup\{\omega(T): T \in P\}$. The substitution $\omega$ can be iterated producing larger and larger patches $\omega^{k}\left(A_{i}\right)$. Notice that $\omega: \mathcal{X}_{\mathcal{A}} \rightarrow \mathcal{X}_{\mathcal{A}}$. The nonnegative matrix $\left[n_{i j}\right]$ is called the substitution matrix. We always assume that $\omega$ is primitive, that is, some power of the substitution matrix is strictly positive. Equivalently, this means that starting with any tile, repeated substitution leads to a patch containing translates of all prototiles.

Definition. A patch is called legal if it is a translate of a subpatch of $\omega^{k}\left(A_{i}\right)$ for some $i \leq m$ and $k \geq 1$. A tiling $T$ is said to be self-affine with the prototile set $\mathcal{A}$, expansion map $\varphi$, and substitution rule $\omega$, if every $\mathcal{T}$-patch is legal. A self-affine tiling is self-similar if $\varphi$ is an expansive similarity.

Warning. There is a large literature on self-similar and self-affine tilings (and tiles), with considerable variation in terminology. We do not attempt to survey it here; the works $[\mathrm{LP}],[\mathrm{T}],[\mathrm{K}],[\mathrm{R} 2],[\mathrm{BG}],[\mathrm{B}],[\mathrm{LW}]$, and [So] give a sample and contain further references.

Many examples of self-similar tilings can be found in [Se] and [G]. Some of the tilings studied in $[\mathrm{T}],[\mathrm{K}],[\mathrm{B}]$, and [So] have a fractal boundary.

The set of self-affine tilings associated with $(\mathcal{A}, \omega)$ is denoted by $\mathcal{X}_{\mathcal{A}, \omega}$. We have $\mathcal{X}_{\mathcal{A}, \omega} \subset \mathcal{X}_{\mathcal{A}}$, and the inclusion is usually proper. It is clear from the definition that $\omega\left(\mathcal{X}_{\mathcal{A}, \omega}\right) \subset \mathcal{X}_{\mathcal{A}, \omega}$. It is well known that, by primitivity of the substitution, $\mathcal{X}_{\mathcal{A}, \omega}$ forms a single $\mathrm{LI}$ class.

Definition. The tilings from $\mathcal{X}_{\mathcal{A}, \omega}$ are said to have the unique composition property if $\omega: \mathcal{X}_{\mathcal{A}, \omega} \rightarrow \mathcal{X}_{\mathcal{A}, \omega}$ is one-to-one.

It is helpful to view the substitution $\omega$ as a composition of two maps: inflation, that is, multiplication by $\varphi$, and subdivision, which is denoted by $S$. To make this precise, we consider "superprototiles" $\varphi A_{i}$ which inherit the marking from $A_{i}$ and on which $\omega$ acts by $\omega\left(\varphi A_{i}\right)=\varphi \omega\left(A_{i}\right)$. Similarly, we define legal "superpatches" and get the set of self-affine supertilings $\mathcal{X}_{\varphi \mathcal{A}, \omega}$. The map $\varphi$ defines a bijection from $\mathcal{X}_{\mathcal{A}, \omega}$ to $\mathcal{X}_{\varphi \mathcal{A}, \omega}$ which is denoted $\varphi$ as well. The subdivision map $S$ decomposes each supertile $\varphi A_{i}+x$ into tiles according to (2), and extends to $S: \mathcal{X}_{\varphi \mathcal{A}, \omega} \rightarrow \mathcal{X}_{\mathcal{A}, \omega}$. Thus, we have $\omega=S \circ \varphi$. Clearly, the unique composition property is equivalent to $S$ being one-to-one.

A "local" equivalent definition of unique composition is often useful. Let $B_{R}(y)$ denote the closed ball of radius $R$ centered at $y$. A self-similar tiling $\mathcal{T}$ has the unique composition property if and only if there exists $R>0$ such that, for any $y \in \mathbf{R}^{d}$ and 
any legal patch covering $B_{R}(y)$, there is a unique way to compose (some of) its tiles into a legal superpatch covering $B_{R^{\prime}-R}(y)$ and with all supertiles intersecting $B_{R^{\prime}-R}(y)$. Equivalence of this property to unique composition is rather straightforward, see Lemma 2.5 for details.

Theorem 1.1. A self-affine tiling has the unique composition property if and only if it is nonperiodic.

Remarks. 1. One direction-that unique composition implies nonperiodicity-is well known, see Chapter 10 of [GS] or Theorem 5.3 of [Se]. Although the set-up in these books is slightly different from ours, the proof remains virtually the same.

2. Baake and Schlottmann [BS] introduced the notion of tilings with inflationdeflation symmetry which seems to generalize self-similar tilings with the unique composition property. A tiling $\mathcal{T}_{2}$ is said to be locally derivable from $\mathcal{T}_{1}$ if there is a translationinvariant local rule which allows tiles of $\mathcal{T}_{2}$ to be recovered if the tiling $\mathcal{T}_{1}$ is known in the neighborhood of some fixed radius. If, in addition, $\mathcal{T}_{1}$ is locally derivable from $\mathcal{T}_{2}$ the two tilings are called mutually locally derivable (MLD). Tilings $\mathcal{T}_{1}$ and $\mathcal{T}_{2}$ are said to belong to the same MLD class if there is a tiling $\mathcal{T}_{2}^{\prime}$ locally isomorphic with $\mathcal{T}_{2}$ and MLD with $\mathcal{T}_{1}$. Finally, a tiling $\mathcal{T}$ is said to have (local) inflation-deflation symmetry with expansion $\varphi$ if $\mathcal{T}$ and $\varphi \mathcal{T}$ belong to the same MLD class. It is plausible that the main result can be generalized to this setting, that is, if $\mathcal{T}$ is locally derivable from some tiling in the LI class of $\varphi \mathcal{T}$, and if $\mathcal{T}$ is nonperiodic, then $\mathcal{T}$ has the inflation-deflation symmetry.

3. Goodman-Strauss [G, Appendix A.7] gave an example showing that unique composition may break down if the original tiling has markings that are absent in the supertiling. We certainly require that if a tile $T \in \mathcal{T}$ has a marking, then this marking is retained for the tile $\varphi T \in \varphi \mathcal{T}$.

4. Strictly speaking, our result applies only to tilings that have superprototiles equal to inflated prototiles. However, it easily extends to, e.g., the kite-and-dart Penrose tiling, since it is MLD with a self-similar Penrose tiling having triangular tiles.

5. Since we assume translational finiteness, tilings such as the Conway-Radin pinwheel tiling [R1] are excluded from consideration; in fact, our methods do not seem to work in that case. The statement (nonperiodicity implies unique composition) might still be true though.

Next we discuss the more general case, when the tiling may have non-trivial periods.

Definition. Let $\mathcal{K}(\mathcal{T})=\left\{x \in \mathbf{R}^{d}: \mathcal{T}+x=\mathcal{T}\right\}$. This is a subgroup of $\mathbf{R}^{d}$ called the translation symmetry group of $\mathcal{T}$. If $\mathcal{K}(\mathcal{T})=\{0\}$, the tiling $\mathcal{T}$ is nonperiodic. If rank $\mathcal{K}(\mathcal{T})=d$, that is, if $\mathcal{K}(\mathcal{T})$ is a lattice, $\mathcal{T}$ is crystallographic.

Clearly, the translation symmetry group is the same for all members of the LI class $\mathcal{X}_{\mathcal{A}, \omega}$. The next result can be interpreted as the "unique composition property modulo $\mathcal{K}$."

Theorem 1.2. Let $\mathcal{K}$ be the translation symmetry group for $\mathcal{X}_{\mathcal{A}, \omega}$. Then, for any two tilings $\mathcal{U}$ and $\mathcal{U}^{\prime}$ in $\mathcal{X}_{\varphi \mathcal{A}, \omega}$,

$$
S \mathcal{U}=S \mathcal{U}^{\prime} \quad \Leftrightarrow \quad \mathcal{U}^{\prime}=\mathcal{U}+g \quad \text { for some } g \in \mathcal{K} .
$$



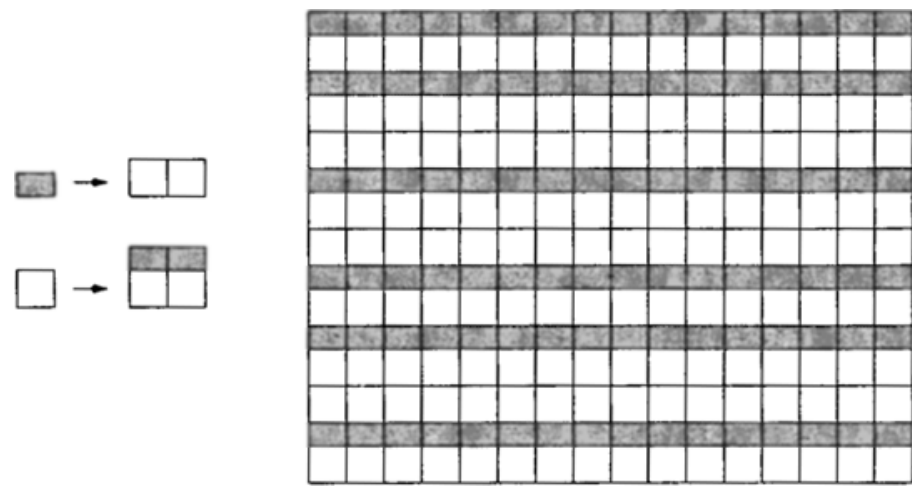

Fig. 2. A self-affine tiling with nontrivial periods.

Remarks. 1 . Theorem 1.1 is the special case of Theorem 1.2 when $\mathcal{K}=\{0\}$.

2. For $x \in \mathcal{K}$ and $\mathcal{T} \in \mathcal{X}_{\mathcal{A}, \omega}$ we have

$$
\omega(\mathcal{T})=\omega(\mathcal{T}-x)=\omega(\mathcal{T})-\varphi x .
$$

Since the translation symmetry group does not depend on a tiling in $\mathcal{X}_{\mathcal{A}, \omega}$ we get that $\varphi \mathcal{K} \subset \mathcal{K}$. Clearly, $\mathcal{K}$ is isomorphic to $\mathbf{Z}^{n}$ for some $0 \leq n \leq d$, and $\varphi \mathcal{K}$ is a subgroup of finite index $[\mathcal{K}: \varphi \mathcal{K}]$. Tilings from $\mathcal{X}_{\varphi \mathcal{A}, \omega}$ have the translation symmetry group $\varphi \mathcal{K}$, so according to Theorem 1.2, the nonuniqueness of composition is measured by $\mathcal{K} / \varphi \mathcal{K}$. The local meaning of unique composition modulo $\mathcal{K}$ is, roughly, that a legal patch can be composed into a legal superpatch in $[\mathcal{K}: \varphi \mathcal{K}]$ essentially distinct ways, at a certain distance from the patch's boundary.

3. The set of tilings $\mathcal{X}_{\mathcal{A}, \omega}$ can be equipped with a metric [RW] making it a compact space. It can be deduced from Theorem 1.2 that $\omega$ is a homeomorphism in the nonperiodic case, and is a covering map with the fiber $\mathcal{K} / \varphi \mathcal{K}$ in general.

Example 3. The tiling in Fig. 2 is self-affine, with the expansion map $\varphi$ dilating by a factor of 2 in the $x$-direction and by a factor of $\frac{1}{2}(1+\sqrt{5})$ in the $y$-direction. There are two prototiles: the square $1 \times 1$ and the shaded rectangle $1 \times \frac{1}{2}(\sqrt{5}-1)$. The prototiles and the substitution rule are indicated in the figure. This tiling is just a product of a trivial $\mathbf{Z}$ periodic one-dimensional tiling and the self-similar one-dimensional "Fibonacci" tiling. The translation symmetry group is $\mathbf{Z} e_{x}$ where $e_{x}$ is the unit vector in the $x$-direction. According to Theorem 1.2, this tiling has the unique composition property modulo $\mathbf{Z} e_{x}$. We have $\varphi \mathbf{Z} e_{x}=2 \mathbf{Z} e_{x}$, so $\left[\mathbf{Z} e_{x}: \varphi \mathbf{Z} e_{x}\right]=2$. Notice that there are a great many ways to compose tiles into supertiles, but if we require the superpatch to be legal, there are just two essentially distinct options.

Theorem 1.2 has implications for the spectral theory of tiling dynamical systems. We mention one result, which improves on Theorem 5.1 of [So]; the proof will appear elsewhere.

Theorem 1.3. All measurable eigenfunctions for the uniquely ergodic dynamical system, arising from the $\mathbf{R}^{d}$-action on $\mathcal{X}_{\mathcal{A}, \omega}$ by translations, can be chosen to be continuous. 
The rest of the paper is organized as follows. Section 2 contains more definitions and several lemmas; some of them may be of independent interest. Then in Section 3 we prove Theorem 1.2 for self-similar tilings. The generalization to self-affine tilings is fairly straightforward but technical. We illustrate the required changes in a key lemma, leaving further details to the reader.

\section{Lemmas}

We introduce some notation and terminology. Consider a tiling $\mathcal{T}$ of $\mathbf{R}^{d}$. For a set $F \subset \mathbf{R}^{d}$, we write

$$
\left.[F]^{\mathcal{T}}=\{T \in \mathcal{T}: T \cap F \neq \emptyset\}, \quad\right] F\left[^{\mathcal{T}}=\{T \in \mathcal{T}: T \subset F\} .\right.
$$

For $T \in \mathcal{T}$ the patch $[T]^{\mathcal{T}}$ is called the $\mathcal{T}$-corona of the tile $\mathcal{T}$. Two tilings $\mathcal{T}_{1}$ and $\mathcal{T}_{2}$ are said to agree on a set $F \subset \mathbf{R}^{d}$ if $[F]^{\mathcal{T}_{1}}=[F]^{\mathcal{T}_{2}}$. The following simple observation is useful:

$$
[F]^{\mathcal{T}}=[F+x]^{\mathcal{T}+x}-x \quad \text { for } \quad x \in \mathbf{R}^{d} .
$$

The next result is well known, see, e.g., [LP].

Lemma 2.1. Let $(\mathcal{A}, \omega)$ be a finite prototile set with a primitive substitution. Then there exist $n_{0} \in \mathbf{N}$ and $\mathcal{T}_{0} \in \mathcal{X}_{\mathcal{A}_{n} \omega}$ such that $\omega^{n_{0}}\left(\mathcal{T}_{0}\right)=\mathcal{T}_{0}$.

We assume that $\omega$ has a fixed point, that is, a tiling $\mathcal{T} \in \mathcal{X}_{\mathcal{A}, \omega}$ such that $\omega(\mathcal{T})=\mathcal{T}$. This does not lead to loss of generality in the nonperiodic case, since we can replace $\omega$ with $\omega^{n_{0}}$ (if $\omega^{n_{0}}$ is one-to-one, then so is $\omega$ ). In the case of nontrivial periods minor adjustments need to be made; they are discussed at the end of the paper.

Observe that the "inflated tiling" $\varphi \mathcal{T}$ has tiles composed of $\mathcal{T}$-tiles (in [K] and [So] and some other papers just the fixed point $\mathcal{T}$ was called self-affine). The tiles of $\varphi^{k} \mathcal{T}$ are called supertiles of order $k$. There is a natural subdivision operation which we denote by $S$. It acts from $\mathcal{X}_{\varphi^{k} \mathcal{A}, \omega}$ to $\mathcal{X}_{\varphi^{k-1}} \mathcal{A}$, $\omega$, as well as on superpatches. We have $S\left(\varphi^{k} \mathcal{T}\right)=\varphi^{k-1} \mathcal{T}$ and $\omega=S \circ \varphi$.

By the definition of a self-similar tiling $\mathcal{U} \in \mathcal{X}_{\mathcal{A}, \omega}$ and by primitivity of $\omega$, every $\mathcal{U}$ patch occurs in some $\omega^{k}\left(A_{1}\right)$, up to translation. It follows that for any $R>0$ there exists $x \in \mathbf{R}^{d}$ such that $\mathcal{T}-x$ agrees with $\mathcal{U}$ on the ball $B_{R}(0)$. We have $\mathcal{T}-x=\omega\left(\mathcal{T}-\varphi^{-1} x\right)$. Letting $R \rightarrow \infty$ and using a diagonalization argument (essentially, compactness of $\mathcal{X}_{\mathcal{A}, \omega}$ in the natural topology), yields that $\omega: \mathcal{X}_{\mathcal{A}, \omega} \rightarrow \mathcal{X}_{\mathcal{A}, \omega}$ is onto [LP], [AP]. Of course, $S=\omega \circ \varphi^{-1}$ is onto as well.

Definition. A tiling $\mathcal{U}$ is said to be repetitive if for any $\mathcal{U}$-patch $P$ there exists $R=R(P)$ such that any ball $B_{R}(y)$ contains a $\mathcal{U}$-patch which is a translate of $P$. We call $\mathcal{U}$ strongly repetitive if we can take $R(P)=C \operatorname{diam}(P)$, with the constant $C$ independent of $P$.

Lemma 2.2. A self-affine tiling $\mathcal{U} \in \mathcal{X}_{\mathcal{A}, \omega}$ is repetitive. 
Proof. The proof is well known but we provide it for completeness. Fix a $\mathcal{U}$-patch $P$. Its translate must occur in some $\omega^{k}\left(A_{1}\right)$. By primitivity, there exists $k_{0} \in \mathbf{N}$ such that $\omega^{k_{0}} A_{i}$ contains a translate of $A_{1}$ for all $i \leq m$. Then the patch $P$ occurs in $\omega^{k+k_{0}} A_{i}$ for all $i$ and so in any supertile of order $k+k_{0}$. Since the decomposition map $S$ is onto, $\mathcal{U}=S^{k+k_{0}} \mathcal{U}^{\prime}$ for some $\mathcal{U}^{\prime} \in \mathcal{X}_{\varphi^{k+k_{0}}} \mathcal{A}, \omega$. For every $\mathcal{U}^{\prime}$-tile, its decomposition $S^{k+k_{0}}$ contains a translate of $P$, and the desired property follows (with $R(P)$ equal to the maximal diameter of a $\mathcal{U}^{\prime}$-tile).

The repetitive property means that every patch occurs in $\mathcal{U}$ with "bounded gaps" (note that repetitivity was called "local isomorphism" in [RW] and [So]). It is equivalent to the minimality of translation action on $\mathcal{X}_{\mathcal{A}, \omega}$ [RW].

Now we restrict ourselves to self-similar tilings, assuming that $\|\varphi(x)\|=\lambda\|x\|$, with $\lambda>1$, and prove that they are strongly repetitive (this, in general, fails for self-affine tilings). Grünbaum and Shephard [GS, Theorem 10.5.4] showed that in the Penrose tiling the minimal distance between two occurrences (translates) of the same patch is bounded by a constant times the diameter of the patch. Their proof essentially establishes strong repetitivity and easily extends to the general case of self-similar tilings.

Lemma 2.3. A self-similar tiling $\mathcal{U} \in \mathcal{X}_{\mathcal{A}, \omega}$ is strongly repetitive: there exists $C>0$ such that for every $\mathcal{U}$-patch $P$, any ball of radius $C \operatorname{diam}(P)$ contains a translate of $P$.

Proof. It is enough to prove that $\mathcal{T}$ is strongly repetitive, where $\omega(\mathcal{T})=\mathcal{T}$. By (1) and the repetitive property, there exists $C_{1}>0$ such that every ball of radius $C_{1}$ contains all $\mathcal{T}$-coronas, up to translation. Again using translation finiteness, $C_{2}>0$ can be found such that, for any $\mathcal{T}$-tiles $T_{1}$ and $T_{2}$,

$$
\operatorname{dist}\left(T_{1}, T_{2}\right)<C_{2} \quad \Rightarrow \quad T_{1} \cap T_{2} \neq \emptyset .
$$

This implies that a set $F \subset \mathbf{R}^{d}$ of diameter less than $C_{2}$ is covered by the $\mathcal{T}$-corona of any tile it meets. Since $\varphi$ is a similarity with expansion constant $\lambda>1$, the same properties hold for the tiling $\varphi^{k} \mathcal{T}$ with $C_{1}$ and $C_{2}$ replaced by $\lambda^{k} C_{1}$ and $\lambda^{k} C_{2}$.

Now let $P$ be a $\mathcal{T}$-patch. Find $k \in \mathbf{N}$ so that $\lambda^{k-1} C_{2} \leq \operatorname{diam}(P)<\lambda^{k} C_{2}$. Then $P$ is covered by some $\varphi^{k} \mathcal{T}$-corona. Every ball of radius $\lambda^{k} C_{1}$ contains a translated copy of this $\varphi^{k} \mathcal{T}$-corona whose $S^{k}$-decomposition contains a translate of $P$ (here we use that $\omega(\mathcal{T})=\mathcal{T}$ so $\left.S^{k}\left(\varphi^{k} \mathcal{T}\right)=\mathcal{T}\right)$. Since

$$
\frac{\lambda^{k} C_{1}}{\operatorname{diam}(P)} \leq \frac{\lambda^{k} C_{1}}{\lambda^{k-1} C_{2}}=\frac{\lambda C_{1}}{C_{2}}=: C
$$

we are done.

An important step in Mossé's proof [M] is the theorem that a nonperiodic primitive substitution is " $N$-power free" for some $N$, that is, it does not contain $v^{N}$ for any word $v$. The tiling analog is that in a nonperiodic self-similar tiling, two occurrences of the same (large) patch cannot be too close to each other. (These patches are allowed to overlap; "not too close" means that they cannot overlap too much.) This contrasts with strong repetitivity. 
Lemma 2.4. Let $\mathcal{U}$ be a self-similar tiling with translation symmetry group $\mathcal{K}$. There exists $N \geq 1$ such that, for any $x, y \in \mathbf{R}^{d}$, if

$$
P \subset \mathcal{U}, \quad P+x \subset \mathcal{U}, \quad B_{r}(y) \subset \operatorname{supp}(P),
$$

then

$$
\|x\| \leq \frac{r}{N} \quad \Rightarrow \quad x \in \mathcal{K} .
$$

In particular, if $\mathcal{U}$ is nonperiodic, then $\|x\| \leq r / N$ implies $x=0$.

Proof. Let $d_{M}$ denote the maximal diameter of a $\mathcal{U}$-tile, and let $\eta>0$ be such that every $\mathcal{U}$-tile contains a ball of diameter $\eta$ in its interior. We prove the statement of the lemma for

$$
N \geq C\left(3 \eta^{-1} d_{M}+1\right),
$$

where $C$ is the strong repetitivity constant from Lemma 2.3 .

Let $P$ be a $\mathcal{U}$-patch such that $P+x \subset \mathcal{U}$ and $\operatorname{supp}(P)$ contains a ball of radius $r$, with $\|x\| \leq r / N$. We need to show that $\mathcal{U}+x=\mathcal{U}$, by the definition of $\mathcal{K}$. Observe that $\mathcal{U}+x=\mathcal{U}$ is equivalent to the implication $T \in \mathcal{U} \Rightarrow T+x \in \mathcal{U}$. Fix a $\mathcal{U}$-tile $T$ and consider the patch

$$
\pi(T)=\{T\} \cup[T+x]^{\mathcal{U}} .
$$

We have $\operatorname{diam}(T \cup(T+x)) \leq\|x\|+d_{M}$, hence $\operatorname{diam}(\pi(T)) \leq\|x\|+3 d_{M}$. By Lemma 2.3 , every ball of radius $C\left(\|x\|+3 d_{M}\right)$ contains a translate of $\pi(T)$. Suppose that $x \neq 0$, otherwise there is nothing to prove. Then $\|x\| \geq \eta$, else having both $P \subset \mathcal{U}$ and $P+x \subset \mathcal{U}$ is impossible (a $\mathcal{U}$-tile and its translate by a vector less that $\eta$ in norm will have intersecting interiors). Then by assumption and (5),

$$
r \geq N\|x\| \geq C\left(3 \eta^{-1} d_{M}+1\right)\|x\| \geq C\left(\|x\|+3 d_{M}\right) .
$$

Since $\operatorname{supp}(P)$ contains a ball of radius $r$, it contains a ball of radius $C\left(\|x\|+3 d_{M}\right)$, hence a translated copy of $\pi(T)$, say, $\pi(T)+g \subset \mathcal{U}$. This patch contains the tile $T^{\prime}=T+g$. We have $T^{\prime} \in P$, so $T^{\prime}+x \in P+x \subset \mathcal{U}$. Moreover, $T^{\prime}+x \in \pi(T)+g$ since

$$
T^{\prime}+x=T+x+g \subset \operatorname{supp}(\pi(T))+g .
$$

It follows that $T+x=\left(T^{\prime}+x\right)-g \in \pi(T) \subset \mathcal{U}$. Since $T$ was an arbitrary $\mathcal{U}$-tile, the proof is finished.

We conclude this section with a restatement of the unique composition property in local terms. It is not used in the paper but, perhaps, helps the reader to understand what unique composition means.

Lemma 2.5. The tiling $\mathcal{T}=\omega(\mathcal{T})$ has the unique composition property if and only if there exists $R>0$ such that, for any $x$ and $y$ in $\mathbf{R}^{d}$,

$$
\left[B_{R}(x)\right]^{\mathcal{T}}=\left[B_{R}(y)\right]^{\mathcal{T}}+(x-y) \quad \Rightarrow \quad\left[B_{1}(x)\right]^{\varphi \mathcal{T}}=\left[B_{1}(y)\right]^{\varphi \mathcal{T}}+(x-y) .
$$

In other words, $\varphi \mathcal{T}$ is locally derivable from $\mathcal{T}$. 
Proof. First we show that (6) implies the unique composition property. Suppose that $S \mathcal{U}=S \mathcal{U}^{\prime}$ for some $\mathcal{U}$ and $\mathcal{U}^{\prime}$ in $\mathcal{X}_{\varphi \mathcal{A}, \omega}$. Choose a sequence $R_{n} \rightarrow \infty$. Since every patch of a tiling from $\mathcal{X}_{\varphi \mathcal{A}, \omega}$ occurs in $\varphi \mathcal{T}$, we can find $x_{n}$ and $y_{n}$ so that $\varphi \mathcal{T}-x_{n}$ and $\varphi \mathcal{T}-y_{n}$ agree with $\mathcal{U}$ and $\mathcal{U}^{\prime}$, respectively, on the ball $B_{R_{n}}(0)$. Then $S\left(\varphi \mathcal{T}-x_{n}\right)=\mathcal{T}-x_{n}$ agrees with $S \mathcal{U}$ and $S\left(\varphi \mathcal{T}-y_{n}\right)=\mathcal{T}-y_{n}$ agrees with $S \mathcal{U}^{\prime}=S \mathcal{U}$ on $B_{R_{n}}(0)$. Now (6) and (4) imply that $\varphi \mathcal{T}-x_{n}$ agrees with $\varphi \mathcal{T}-y_{n}$ on $B_{R_{n}-R}(0)$, hence $\mathcal{U}$ agrees with $\mathcal{U}^{\prime}$ on $B_{R_{n}-R}(0)$. Letting $n \rightarrow \infty$ yields $\mathcal{U}=\mathcal{U}^{\prime}$, as desired.

Now we prove the converse. Suppose that (6) does not hold. Then, by (4), there exist $R_{n} \rightarrow \infty$ and $x_{n}, y_{n} \in \mathbf{R}^{d}$ such that $\mathcal{T}-x_{n}$ agrees with $\mathcal{T}-y_{n}$ on $B_{R_{n}}(0)$ but $\varphi \mathcal{T}-x_{n}$ does not agree with $\varphi \mathcal{T}-y_{n}$ on $B_{1}(0)$. Using a diagonalization argument, we can assume that $\varphi \mathcal{T}-x_{n}$ and $\varphi \mathcal{T}-y_{n}$ "converge," respectively, to some tilings $\mathcal{U}$ and $\mathcal{U}^{\prime}$ in $\mathcal{X}_{\varphi, \mathcal{A}, \omega}$. Since $S\left(\varphi \mathcal{T}-x_{n}\right)=\mathcal{T}-x_{n}$ and $S\left(\varphi \mathcal{T}-y_{n}\right)=\mathcal{T}-y_{n}$ agree on $B_{R_{n}}(0)$, it follows that $S \mathcal{U}=S \mathcal{U}^{\prime}$. On the other hand, $\varphi \mathcal{T}-x_{n}$ does not agree with $\varphi \mathcal{T}-y_{n}$ on $B_{1}(0)$, so $\mathcal{U} \neq \mathcal{U}^{\prime}$, a contradiction with the unique composition property.

\section{Proof of the Main Theorem}

Here we give a proof of Theorem 1.2 assuming that $\omega$ has a fixed point and that the expansion map $\varphi$ is self-similar. At the end of the section we indicate the changes needed to deal with the general case. We emphasize once again that the proof is modeled after $[\mathrm{M}]$.

Let $\mathcal{K}$ be the translation symmetry group for $\mathcal{X}_{\mathcal{A}, \omega}$. Suppose that $\mathcal{T}$ is a self-affine tiling with expansion map $\varphi$, satisfying $\omega(\mathcal{T})=\mathcal{T}$. If $\mathcal{U} \in \mathcal{X}_{\varphi \mathcal{A}, \omega}$ and $\mathcal{U}^{\prime}=\mathcal{U}+g$ for some $g \in \mathcal{K}$, then $S \mathcal{U}^{\prime}=S \mathcal{U}+g=S \mathcal{U}$ since $S \mathcal{U} \in \mathcal{X}_{\mathcal{A}, \omega}$. This proves the easy implication of Theorem 1.2.

Now we turn to the converse. Recall that $\varphi \mathcal{K} \subset \mathcal{K}$ and the quotient $\mathcal{K} / \varphi \mathcal{K}$ is finite; let $\Gamma \subset \mathcal{K}$ be a complete family of coset representatives $\bmod \varphi \mathcal{K}$.

Lemma 3.1. Suppose that there exists $r>0$ such that, for any $R>r$, if $\mathcal{T}-x$ agrees with $\mathcal{T}-y$ on $B_{R}(0)$, then $\varphi \mathcal{T}-x$ agrees with $\varphi \mathcal{T}-y-g$ on $B_{R / 2}(0)$ for some $g \in \Gamma$. Then $\mathcal{T}$ satisfies (3).

Proof. The proof is required only for the implication " $\Rightarrow$ " in (3). It is analogous to the corresponding part of Lemma 2.5 .

Suppose that $S \mathcal{U}=S \mathcal{U}^{\prime}$ for two tilings $\mathcal{U}$ and $\mathcal{U}^{\prime}$ in $\mathcal{X}_{\varphi \mathcal{A}, \omega}$. We want to show that $\mathcal{U}^{\prime}=\mathcal{U}+g$ for some $g \in \mathcal{K}$. Choose a sequence $R_{n} \rightarrow \infty$. We can find $x_{n}, y_{n} \in \mathbf{R}^{d}$ so that $\varphi \mathcal{T}-x_{n}$ and $\varphi \mathcal{T}-y_{n}$ agree with $\mathcal{U}$ and $\mathcal{U}^{\prime}$, respectively, on the ball $B_{R_{n}}(0)$. Then $S\left(\varphi \mathcal{T}-x_{n}\right)=\mathcal{T}-x_{n}$ and $S\left(\varphi \mathcal{T}-y_{n}\right)=\mathcal{T}-y_{n}$ agree with $S \mathcal{U}=S \mathcal{U}^{\prime}$ on $B_{R_{n}}(0)$. By assumption, $\varphi \mathcal{T}-x_{n}$ agrees with $\varphi \mathcal{T}-y_{n}-g_{n}$ on $B_{R_{n} / 2}(0)$ for some $g_{n} \in \Gamma$. Since $\Gamma$ is a finite set, passing to a subsequence, it can be assumed that $g_{n}=g=$ const. Since $\varphi \mathcal{T}-y_{n}$ agrees with $\mathcal{U}^{\prime}$ on $B_{R_{n}}(0)$, the tiling $\varphi \mathcal{T}-y_{n}-g$ agrees with $\mathcal{U}^{\prime}-g$ on $B_{R_{n} / 2}(0)$, as long as $R_{n} / 2>\|g\|$. Thus, $\mathcal{U}$ agrees with $\mathcal{U}^{\prime}-g$ on $B_{R_{n} / 2}(0)$ for $n$ sufficiently large. Letting $n \rightarrow \infty$ yields $\mathcal{U}=\mathcal{U}^{\prime}-g$, as desired. 
Proof of Theorem 1.2. Suppose that (3) does not hold. Then, by Lemma 3.1 and (4), $R>0$, arbitrarily large, and $x, y \in \mathbf{R}^{d}$ can be found such that

$$
\left[B_{R}(x)\right]^{\mathcal{T}}=\left[B_{R}(y)\right]^{T}+(x-y),
$$

but

$$
\varphi \mathcal{T}-x \text { does not agree with } \varphi \mathcal{T} x-y-g \text { on } B_{R / 2}(0) \quad \text { for any } g \in \Gamma \text {. (8) }
$$

Now we assume that $\varphi$ is self-similar: $\|\varphi x\|=\lambda\|x\|$ for all $x \in \mathbf{R}^{d}$. Let $k=k(R) \in \mathbf{N}$ be such that

$$
\lambda^{k}(2 N+6) d_{M} \leq R<\lambda^{k+1}(2 N+6) d_{M},
$$

where $N \geq 1$ comes from Lemma 2.4 and $d_{M}$ is the maximal diameter of a $\mathcal{T}$-tile. Consider $] B_{R}(x)\left[{ }^{\varphi^{k}} \mathcal{T}\right.$, the collection of supertiles of order $k$ contained in $B_{R}(x)$. We have

$$
] B_{R}(x)\left[\varphi^{\varphi^{k}} \mathcal{T}=\varphi^{k} E \quad \text { for some } \quad E \subset \mathcal{T}\right.
$$

Similarly,

$$
] B_{R-\lambda^{k} d_{M}}(y)\left[^{\varphi^{k} \mathcal{T}}=\varphi^{k} F \quad \text { for some } \quad F \subset \mathcal{T} .\right.
$$

Since $\lambda^{k} d_{M}=\max \left\{\operatorname{diam}\left(\varphi^{k} T\right): T \in \mathcal{T}\right\}$, it is clear that

$$
\operatorname{supp}\left(\varphi^{k} E\right) \supset B_{R-\lambda^{k} d_{M}}(x), \quad \operatorname{supp}\left(\varphi^{k} F\right) \supset B_{R-2 \lambda^{k} d_{M}}(y) .
$$

Observe that $\lambda^{k} \operatorname{diam}(E)=\operatorname{diam}\left(\varphi^{k} E\right) \leq 2 R$, so, by (9), $\operatorname{diam}(E)$ is bounded by a constant independent of $R$. The same bound holds for $\operatorname{diam}(F)$. Condition (1) implies that there are finitely many possibilities for $E$ and $F$, up to translation.

Now we repeat the whole construction for $R_{n} \rightarrow \infty$, yielding $x_{n}, y_{n}, k_{n}, E_{n}$, and $F_{n}$. There exist $i$ and $j$ such that $E_{j}$ is a translate of $E_{i}$ and $F_{j}$ is a translate of $F_{i}$, with $R_{j}>\lambda R_{i}$. To simplify notation, we let

$$
(x, y, R, k, E, F):=\left(x_{i}, y_{i}, R_{i}, k_{i}, E_{i}, F_{i}\right)
$$

and

$$
\left(x^{\prime}, y^{\prime}, R^{\prime}, k^{\prime}, E^{\prime}, F^{\prime}\right):=\left(x_{j}, y_{j}, R_{j}, k_{j}, E_{j}, F_{j}\right) .
$$

The choice of $i$ and $j$ implies that $E^{\prime}=E+z_{1}$ and $F^{\prime}=F+z_{2}$, for some $z_{1}$ and $z_{2}$ in $\mathbf{R}^{d}$. Let $l:=k^{\prime}-k$. The analog of $(9)$ is

$$
\lambda^{k+l}(2 N+6) d_{M} \leq R^{\prime}<\lambda^{k+l+1}(2 N+6) d_{M} .
$$

Since $R^{\prime}>\lambda R$, we have $l \geq 1$. The analogs of (7) and (8) for $x^{\prime}, y^{\prime}, R^{\prime}$ are referred to as $\left(7^{\prime}\right)$ and $\left(8^{\prime}\right)$. The situation can be summarized as follows:

$$
\begin{aligned}
] B_{R}(x)\left[\varphi^{\varphi^{k} T}\right. & =\varphi^{k} E, & ] B_{R-\lambda^{k} d_{M}}(y)\left[^{\varphi^{k} T}\right. & =\varphi^{k} F, \\
] B_{R^{\prime}}\left(x^{\prime}\right)\left[^{\varphi^{k+l} \mathcal{T}}\right. & =\varphi^{k+l}\left(E+z_{1}\right), & ] B_{R^{\prime}-\lambda^{k+l} d_{M}}\left(y^{\prime}\right)\left[^{\varphi^{k+l} \mathcal{T}}\right. & =\varphi^{k+l}\left(F+z_{2}\right) .
\end{aligned}
$$


Let

and

$$
Q_{1}:=S^{k} \varphi^{k+l} F+\varphi^{l}(x-y)+\varphi^{k+l} x_{1}, \quad P_{1}:=S^{l} Q_{1}
$$

$$
Q_{2}:=S^{k} \varphi^{k+l} F+\varphi^{k+l} z_{2}+\left(x^{\prime}-y^{\prime}\right), \quad P_{2}:=S^{l} Q_{2}
$$

Now we make two claims about the patches $P_{1}, P_{2}, Q_{1}$, and $Q_{2}$, finish the argument assuming that they are true, and then prove the claims.

Claim 1. We have

and

$$
\left.P_{1} \subset\right] B_{R^{\prime}}\left(x^{\prime}\right)\left[^{T}\right.
$$

$$
\left.\left[B_{R^{\prime}-2 \lambda^{k+l} d_{M}}\left(x^{\prime}\right)\right]^{\mathcal{T}} \subset P_{2} \subset\right] B_{R^{\prime}-\lambda^{k+1} d_{M}}\left(x^{\prime}\right)\left[^{\mathcal{T}} .\right.
$$

It is clear from (11) and (12) that $P_{2}=P_{1}+h$ and $Q_{2}=Q_{1}+h$ where

$$
h=\varphi^{k+l} z_{2}+\left(x^{\prime}-y^{\prime}\right)-\varphi^{l}(x-y)-\varphi^{k+l} z_{1} \text {. }
$$

Assuming Claim 1, we conclude from (13) and (14) that

$$
B_{R^{\prime}-2 \lambda^{k+l} d_{M}}\left(x^{\prime}\right)+h \subset B_{R^{\prime}}\left(x^{\prime}\right)
$$

hence

$$
\|h\| \leq 2 \lambda^{k+l} d_{M}
$$

We can apply Lemma 2.4 since $P_{2} \subset \mathcal{T}$ and $P_{2}-h=P_{1} \subset \mathcal{T}$, the support of the patch $P_{2}$ contains a ball of radius $R^{\prime}-2 \lambda^{k+l} d_{M}$ by (14), and

$$
\|h\| \leq 2 \lambda^{k+l} d_{M} \leq\left(R^{\prime}-2 \lambda^{k+l} d_{M}\right) / N
$$

by (10). Thus, $h \in \mathcal{K}$.

Claim 2. We have

and

$$
Q_{1} \supset\left[B_{R^{\prime} / 2}\left(x^{\prime}\right)\right]^{\varphi^{\prime} \mathcal{T}}
$$

$$
Q_{2}+\left(y^{\prime}-x^{\prime}\right) \supset\left[B_{R^{\prime} / 2}\left(y^{\prime}+h\right)\right]^{\varphi^{\prime} T} \text {. }
$$

Assuming Claim 2, we obtain

$$
\begin{aligned}
{\left[B_{R^{\prime} / 2}\left(x^{\prime}\right)\right]^{\varphi} \mathcal{T} \subset S^{l-1} Q_{1} } & =\left[S^{l-1} Q_{2}+\left(y^{\prime}-x^{\prime}\right)\right]+\left(x^{\prime}-y^{\prime}\right)-h \\
& \supset\left[B_{R^{\prime} / 2}\left(y^{\prime}\right)\right]^{\varphi T}+\left(x^{\prime}-y^{\prime}\right)-h \\
& =\left[B_{R^{\prime} / 2}\left(x^{\prime}\right)\right]^{\varphi \mathcal{T}+x^{\prime}-y^{\prime}-h}
\end{aligned}
$$

using (4) in the last equality. This implies that $\varphi \mathcal{T}-x^{\prime}$ agrees with $\varphi \mathcal{T}-y^{\prime}-h$ on $\left[B_{R^{\prime} / 2}(0)\right]$. Since $\Gamma$ contains all coset representatives $\bmod \varphi \mathcal{K}$, there exists $g \in \Gamma$ such that $h-g \in \varphi \mathcal{K}$. Then $\varphi \mathcal{T}-y^{\prime}-g=\varphi \mathcal{T}-y^{\prime}-h$ agrees with $\varphi \mathcal{T}-x^{\prime}$ on $B_{R^{\prime} / 2}(0)$. This is a contradiction with $\left(8^{\prime}\right)$.

To complete the proof it remains to verify Claims 1 and 2 . 
Proof of Claims 1 and 2. We often use the following simple facts:

$$
\left.S[F]^{\varphi \mathcal{T}} \supset[F]^{\mathcal{T}} \text { and } S\right] F\left[^{\varphi \mathcal{T}} \subset\right] F\left[^{\mathcal{T}}\right. \text {. }
$$

We have $] B_{R-\lambda^{k} d_{M}}(y)\left[\varphi^{\varphi^{k} T}=\varphi^{k} F\right.$, hence $\left.S^{k} \varphi^{k} F \subset\right] B_{R-\lambda^{k} d_{M}}(y)\left[{ }^{T}\right.$. By (7),

$$
\begin{aligned}
S^{k} \varphi^{k} F+(x-y) & \subset] B_{R-\lambda^{k} d_{M}}(x)\left[{ }^{\mathcal{T}}\right. \\
& \subset\left[B_{R-\lambda^{k} d_{M}}(x)\right]^{\mathcal{T}} \\
& \subset S^{k}\left[B_{R-\lambda^{k} d_{M}}(x)\right]^{\varphi^{k} \mathcal{T}} \\
& \subset] B_{R}(x)\left[^{\varphi^{k} \mathcal{T}}=S^{k} \varphi^{k} E .\right.
\end{aligned}
$$

Hence

$$
\begin{aligned}
Q_{1}=\varphi^{l}\left(S^{k} \varphi^{k} F+(x-y)\right)+\varphi^{k+l} z_{1} & \subset \varphi^{l} S^{k} \varphi^{k} E+\varphi^{k+l} z_{1} \\
& =S^{k}\left(\varphi^{k+l}\left(E+z_{1}\right)\right) \\
& \left.=S^{k}\right] B_{R^{\prime}}\left(x^{\prime}\right)\left[^{\varphi^{k+l}} \mathcal{T}\right. \\
& \subset] B_{R^{\prime}}\left(x^{\prime}\right)\left[\varphi^{\prime} \mathcal{T} .\right.
\end{aligned}
$$

It follows that $\left.P_{1}=S^{l} Q_{1} \subset\right] B_{R^{\prime}}\left(x^{\prime}\right)\left[^{\mathcal{T}}\right.$, and (13) is verified. Next,

$$
\left.\varphi^{k+l} F+\varphi^{k+l} z_{2}=\right] B_{R^{\prime}-\lambda^{k+l} d_{M}}\left(y^{\prime}\right)\left[^{\varphi^{k+1} \mathcal{T}},\right.
$$

therefore,

$$
\left.\left[B_{R^{\prime}-2 \lambda^{k+l} d_{M}}\left(y^{\prime}\right)\right]^{\varphi^{\prime} \mathcal{T}} \subset S^{k} \varphi^{k+l} F+\varphi^{k+l} z_{2} \subset\right] B_{R^{\prime}-\lambda^{k+l} d_{M}}\left(y^{\prime}\right)\left[^{\varphi^{\prime} \mathcal{T}} .\right.
$$

Applying $S^{\prime}$ we obtain

$$
\left.\left[B_{R^{\prime}-2 \lambda^{k+1} d_{M}}\left(y^{\prime}\right)\right]^{\mathcal{T}} \subset S^{k+l} \varphi^{k+l} F+\varphi^{k+l} z_{2} \subset\right] B_{R^{\prime}-\lambda^{k+l} d_{M}}\left(y^{\prime}\right)\left[^{T} .\right.
$$

By $\left.\left(7^{\prime}\right),\right] B_{R^{\prime}}\left(y^{\prime}\right)\left[{ }^{\mathcal{T}}+\left(x^{\prime}-y^{\prime}\right)=\right] B_{R^{\prime}}\left(x^{\prime}\right)\left[^{\mathcal{T}}\right.$, so translating $(20)$ by $\left(x^{\prime}-y^{\prime}\right)$ yields $(14)$.

Now we turn to Claim 2. Recall (18) that $Q_{1} \subset 1 B_{R^{\prime}}\left(x^{\prime}\right)\left[\varphi^{\prime} \mathcal{T}\right.$, so to prove (16) it remains to check that $\operatorname{supp}\left(Q_{1}\right) \supset B_{R^{\prime} / 2}\left(x^{\prime}\right)$. However,

$$
\operatorname{supp}\left(Q_{1}\right)=\operatorname{supp}\left(P_{1}\right)=\operatorname{supp}\left(P_{2}\right)-h \supset B_{R^{\prime}-2 \lambda^{k+1} d_{M}}\left(x^{\prime}\right)-h \supset B_{R^{\prime}-4 \lambda^{k+1} d_{M}}\left(x^{\prime}\right),
$$

by (14) and (15). Since $R^{\prime}-4 \lambda^{k+l} d_{M} \geq R^{\prime} / 2$ by (10), the inclusion (16) follows. Finally, (17) is implied by the left inclusion in (19) and the inequality $R^{\prime}-2 \lambda^{k+1} d_{M}$ $\geq R^{\prime} / 2+h$.

Both Claims 1 and 2 have been verified, and the proof of the theorem is finished.

Self-Affine Tilings. The scheme of the proof of Theorem 1.2 remains the same, but instead of the balls $B_{R}(0)$, we have to work with sets of the form $\varphi^{k} V$ for some fixed neighborhood of the origin $V$. We do not repeat the whole proof; rather, the required changes are illustrated by proving the analog of Lemma 2.4 .

Fix $V$, a ball centered at the origin, such that every tile contains a translate of $V$ in its interior. 
Lemma 3.2. Let $\mathcal{T}$ be a self-affine tiling with translation symmetry group $\mathcal{K}$ and expansion map $\varphi$. There exists $N \in \mathbf{N}$ such that, for any $l \in \mathbf{N}$ and $x, y \in \mathbf{R}^{d}$, if

$$
P \subset \mathcal{T}, \quad P+x \subset \mathcal{T}, \quad \varphi^{l} V+y \subset \operatorname{supp}(P),
$$

then

$$
x \in \varphi^{l-N} V \quad \Rightarrow \quad x \in \mathcal{K}
$$

Proof. As above, we assume that $\omega(\mathcal{T})=\mathcal{T}$. Positive integers $n_{1}, n_{2}, n_{3}$ with the following properties can be found:

(a) For any $T \in \mathcal{T}$ and any $z \in T$,

$$
T \subset z+\varphi^{n_{1}} V .
$$

(b) For any $z \in \mathbf{R}^{d}$ there exists a $\mathcal{T}$-corona $E$ such that

$$
V+z \subset \operatorname{supp}\left(\varphi^{n_{2}} E\right) \text {. }
$$

(c) For any $z \in \mathbf{R}^{d}$, the set $z+\varphi^{n_{3}} V$ contains all $\mathcal{T}$-coronas, up to translation.

Notice that (c) holds by the repetitive property which was proved for self-affine tilings (Lemma 2.2). Applying the map $\varphi^{k}$ in (a)-(c), we obtain analogous properties for $\varphi^{k} \mathcal{T}$ tiles and patches.

Recall that $\|\varphi x\| \geq \lambda_{0}\|x\|$, with $\lambda_{0}>1$. Let $s$ be such that $\lambda_{0}^{s} \geq 2$. We claim that the statement of the lemma holds with $N=n_{1}+n_{2}+n_{3}+s$.

Suppose that $P \subset \mathcal{T}, P+x \subset \mathcal{T}, \varphi^{l} V+y \subset \operatorname{supp}(P)$, and $x \in \varphi^{l-N} V$. We want to show that $\mathcal{T}+x=\mathcal{T}$, that is, for any $\mathcal{T}$-tile $T$, its translate $T+x$ is again a $\mathcal{T}$-tile. It can be assumed that $l>N$ since a translate of any tile by a vector in $V$ will have an interior intersecting the tile itself. Pick any tile $T$ and a point $c(T) \in T$. Consider the $\mathcal{T}$-patch

$$
\pi(T)=\{T\} \cup[c(T)+x]^{\mathcal{T}}
$$

We have

$$
\operatorname{supp}(\pi(T)) \subset\left(c(T)+\varphi^{n_{1}} V\right) \cup\left(c(T)+\varphi^{l-N} V+\varphi^{n_{1}} V\right) \subset c(T)+\varphi^{l-N} V+\varphi^{n_{1}} V
$$

Observe that $V \subset \varphi^{i} V$ for $i \geq 0$, hence $\varphi^{i} V \subset \varphi^{j} V$ for $i \leq j$, so

$$
\varphi^{i} V+\varphi^{j} V \subset 2 \varphi^{i+j} V \subset \varphi^{i+j+s} V .
$$

Thus,

$$
\operatorname{supp}(\pi(T)) \subset c(T)+\varphi^{l+n_{1}+s-N} V .
$$

Now we use property (b) to find a $\mathcal{T}$-corona $E$ such that

$$
\operatorname{supp}(\pi(T)) \subset \operatorname{supp}\left(\varphi^{l+n_{1}+n_{2}+s-N} E\right)=\operatorname{supp}\left(\varphi^{l-n_{3}} E\right) .
$$

By assumption, $\varphi^{l} V+y \subset \operatorname{supp}(P)$, so by property (c), $\operatorname{supp}(P)$ contains all $\varphi^{l-n_{3}} \mathcal{T}$ coronas, up to translation. It follows that $P$ contains a translate of $\pi(T)$ as a subpatch, say, $\pi(T)-g \subset P$. Then $T-g \in P$ and $T^{\prime}=T-g+x \in P$. Further, $(T+x) \cap \operatorname{supp}(\pi(T)) \neq$ $\emptyset$, hence

$$
T+x=T^{\prime}+g \in \pi(T) \subset \mathcal{T} \text {. }
$$

The proof is complete. 
When $\omega$ Has No Fixed Points. All the proofs in Sections 2 and 3 readily extend to this case. We still have that $\omega$ and $S$ are onto. Instead of the tiling $\mathcal{T}$, satisfying $\omega(\mathcal{T})=\mathcal{T}$, and $\varphi^{k} \mathcal{T}$, we can work with an arbitrary tiling $\mathcal{U}=\mathcal{U}_{0} \in \mathcal{X}_{\mathcal{A}, \omega}$ and any sequence of tilings $\mathcal{U}_{k} \in \mathcal{X}_{\varphi^{k}} \mathcal{A}_{, \omega}$ satisfying $S \mathcal{U}_{k}=\mathcal{U}_{k-1}$, for $k \geq 1$. The details are straightforward.

\section{Acknowledgments}

I am grateful to C. Skau, M. Baake, and M. Schlottmann for helpful discussions. I also thank the anonymous referees for several valuable suggestions.

\section{References}

[AP] J. Anderson and I. Putnam, Topological invariants for substitution tilings and their associated $C^{*}$ algebras, Ergodic Theory Dynamical Systems, to appear.

[BS] M. Baake and M. Schlottmann, Geometric aspects of tilings and equivalence concepts, in Proc. ICQ5. World Scientific, Singapore, 1995, pp. 15-21.

[B] C. Bandt, Self-similar tilings and patterns described by mappings, in The Mathematics of Aperiodic Order-Proceedings of NATO-Advanced Studies Institute, Waterloo, ON, August 1995, to appear.

[BG] C. Bande and G. Geibrich, Classification of self-affine lattice tilings, J. London Math. Soc. 50:2 (1994), 581-593.

[G] C. Goodman-Strauss, Matching rules and substitution tilings, Preprint, 1996.

[GS] B. Grünbaum and G.S. Shephard, Tilings and Patterns, Freeman, New York, 1986.

[K] R. Kenyon, The construction of self-similar tilings, Geom. Funct. Anal. 6:3 (1996), 471 488 .

[LW] J. C. Lagarias and Y. Wang, Self-affine tiles in $\mathbf{R}^{n}$, Adv. in Math 121:1 (1996), 21-49.

[LP] W. F. Lunnon and P. A. B. Pleasants, Quasicrystallographic tilings, J. Math. Pures Appl. 66 (1987), 217-263.

[M] B. Mossé, Puissances de mots et reconnaisabilité des point fixes d'une substitution, Theoret Comput. Sci. 99:2 (1992), 327-334.

[R1] C. Radin, The pinwheel tiling of the plane, Ann. of Math. 139 (1994), 661-702.

[R2] C. Radin, Space tilings and substitutions, Geom. Dedicata 55 (1995), 257-264.

[RW] C. Radin and M. Wolff, Space tilings and local isomorphism, Geom. Dedicata 42 (1992), 355-360.

[Se] M. Senechal, Quasicrystals and Geometry. Cambridge University Press, Cambridge, 1995.

[So] B. Solomyak, Dynamics of self-similar tilings, Ergodic Theory Dynamical Systems, to appear.

[T] W. Thurston, Groups, Tilings, and Finite State Automata, AMS Colloquium Lecture Notes, American Mathematical Society, Boulder, 1989.

Received August 7, 1996, and in revised form December 18, 1996. 